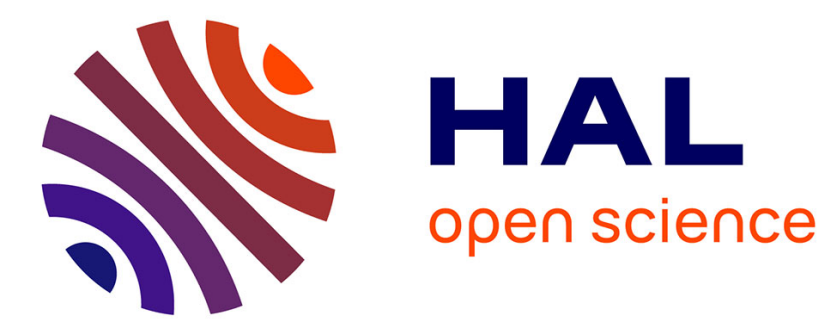

\title{
Intersection Graphs of Jordan Arcs
}

Hubert de Fraysseix, Patrice Ossona de Mendez

\section{To cite this version:}

Hubert de Fraysseix, Patrice Ossona de Mendez. Intersection Graphs of Jordan Arcs. Discrete Mathematics and Theoretical Computer Science, 1999, 49, pp.11-28. hal-00005625

\section{HAL Id: hal-00005625 \\ https://hal.science/hal-00005625}

Submitted on 18 Aug 2005

HAL is a multi-disciplinary open access archive for the deposit and dissemination of scientific research documents, whether they are published or not. The documents may come from teaching and research institutions in France or abroad, or from public or private research centers.
L'archive ouverte pluridisciplinaire HAL, est destinée au dépôt et à la diffusion de documents scientifiques de niveau recherche, publiés ou non, émanant des établissements d'enseignement et de recherche français ou étrangers, des laboratoires publics ou privés. 
DIMACS Series in Discrete Mathematics

and Theoretical Computer Science

\title{
Intersection Graphs of Jordan Arcs
}

\author{
P. Ossona de Mendez and H. de Fraysseix
}

\begin{abstract}
A family of Jordan arcs, such that two arcs are nowhere tangent, defines a hypergraph whose vertices are the arcs and whose edges are the intersection points. We shall say that the hypergraph has a strong intersection representation and, if each intersection point is interior to at most one arc, we shall say that the hypergraph has a strong contact representation.

We first characterize those hypergraphs which have a strong contact representation and deduce some sufficient conditions for a simple planar graph to have a strong intersection representation.

Then, using the Four Color Theorem, we prove that a large class of simple planar graphs have a strong intersection representation.
\end{abstract}

\section{Introduction}

A family of Jordan arcs, such that two arcs are nowhere tangent, defines a hypergraph whose vertices are the arcs and whose edges are the intersection points. We shall say that the hypergraph has a strong intersection representation and, if each intersection point is interior to at most one arc, we shall say that the hypergraph has a strong contact representation.

Such a family classically defines a string graph, which is a simple graph, whose vertices are the arcs, two vertices being adjacent if the corresponding arcs intersect at least once [3]. It is not known whether deciding whether a graph is a string graph is algorithmically decidable [11]. However, all planar graphs are obviously string graphs.

If two arcs may only intersect once, we shall say that the intersection graph has an intersection representation. Deciding whether a given graph has an intersection representation is known to belong to the NP-complete class [9], even if strong restrictions are requested on the family of arcs [10]. It has been a challenge for long to prove or disprove that all planar graphs have an intersection representation. It is clear that a simple graph has an intersection representation if and only if it has a strong intersection representation.

First, we shall introduce a general framework on strong contact representation.

Then, the introduction of a 4-coloration will lead to prove that a large class of planar graphs have an intersection representation.

Most of the results proved here were first presented in [5] and [7].

This work was partially supported by the Esprit LTR Project no 20244-ALCOM IT. 


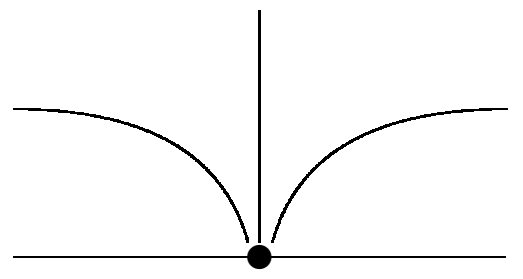

FiguRE 1. Locally one-sided contact
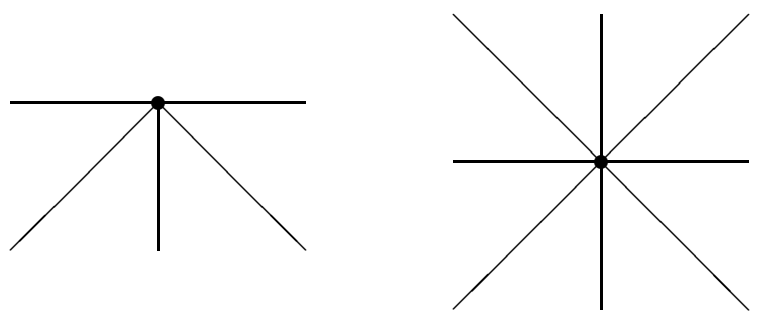

FIGURE 2. A contact and an intersection representations of $K_{4}$

\section{General Framework}

Arc intersection problems arise topological difficulties that vanish when arcs are only in contact, as the arc-point incidence then defines a plane graph (i.e. a planar graph together with an embedding).

A contact family $\mathcal{F}=(\mathcal{V}, \mathcal{P})$ of Jordan arcs is a finite family $\mathcal{V}$ of Jordan $\operatorname{arcs}$ in the plane and a finite point set $\mathcal{P}$ on these arcs, such that two arcs share at most one point (called contact point), and such that all the contact points belong to $\mathcal{P}$ and are interior to at most one arc. Furthermore, at each point $p$ interior to an $\operatorname{arc} \nu$, the $\operatorname{arc} \nu$ appears twice consecutively in a clockwise traversal around $p$ (i.e. we consider only locally one-sided contacts). This family defines a particular type of intersection graph, called contact graph (which may be not planar) and also a colored planar bipartite arc-point incidence $\operatorname{graph} \operatorname{Incid}(\mathcal{F})$, whose vertex set is the union of the arc set $\mathcal{V}$ (colored white) and the contact point set $\mathcal{P}$ (colored black), and whose incidence is the inclusion relation of a point in an arc.

\section{Graphs, Hypergraphs and Frames}

2.1. Graphs. Given a graph $G$, we use the following notations:

- $V(G)$ is the vertex set of $G$,

- $E(G)$ is the edge set of $G$,

- $N(x)$ is the neighbor set of the vertex $x$,

- $N(A)=\bigcup_{x \in A} N(x)$ is the neighbor set of a subset $A$ of vertices,

- $d_{G}(x)$ is the degree of the vertex $x$,

- $G_{A}$ is the subgraph induced by the subset $A$ of vertices,

- $T(G)$ is the set of bounded triangular faces of $G$, if $G$ is a plane graph (i.e. a planar graph embedded in the plane). 

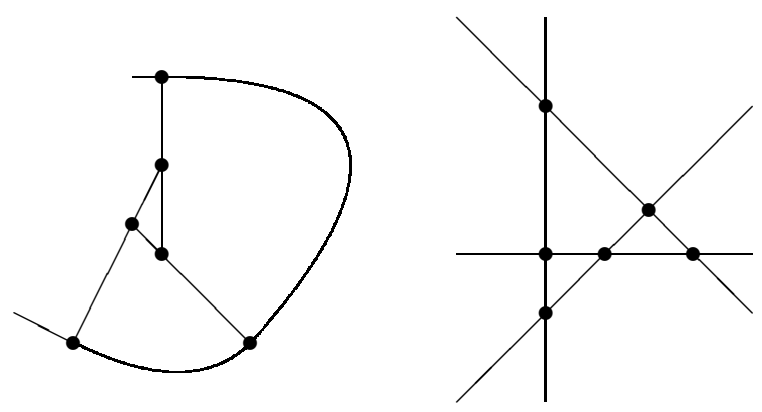

FIgURE 3. A strong contact and a strong intersection representations of $K_{4}$
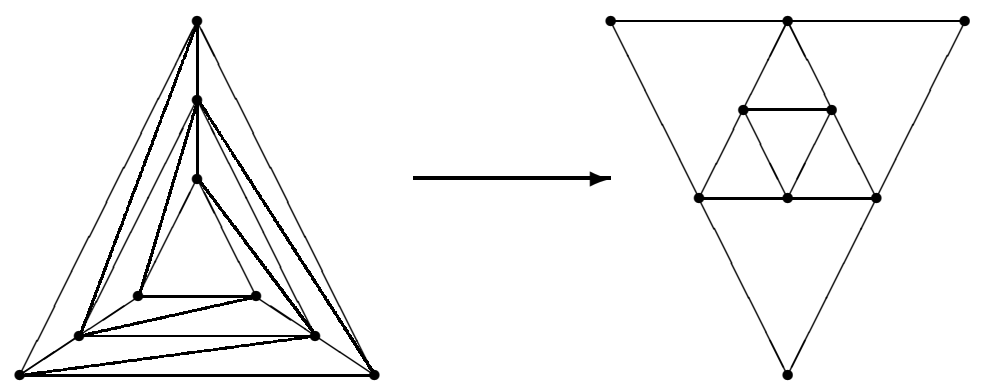

FIGURE 4. A planar graph, which has a contact representation, but no strong contact representation

DeFINITION 2.1. A graph $G$ has a strong contact representation if there exists a contact family $\mathcal{F}$ such that the arc-point incidence of $\mathcal{F}$ is the vertex-edge incidence of $G$

REMARK 2.2. In a strong contact representation, the points represent the edges and thus belong to exactly 2 arcs, unlike the contact points of a contact representation which represent cliques.

2.2. Hypergraphs. A hypergraph is a pair $\mathcal{H}=(X, \mathcal{E})$, where $X$ is a finite set and $\mathcal{E}$ is a family $\left(E_{i}, i \in I\right)$ of subsets of $X$, such that: $E_{i} \neq \emptyset(\forall i \in I)$ and $\bigcup_{i \in I} E_{i}=X$. The elements of $X$ and $\mathcal{E}$ are respectively the vertices and the edges of the hypergraph. Two vertices $x, y \in X$ are adjacent if they both belong to some edge of $\mathcal{H}$; two edges $E_{i}, E_{j}$ are adjacent if their intersection is not empty. A vertex $x \in X$ is incident to an edge $E_{i} \in \mathcal{E}$ if $x$ belongs to $E_{i}$ (see 近).

A hypergraph $\mathcal{H}$ is linear if any two edges have at most one common element:

$$
\forall i \neq j, \quad\left|E_{i} \cap E_{j}\right| \leq 1
$$

The sub-hypergraph of $\mathcal{H}$ induced by a subset $Y \subseteq X$ is the hypergraph $\mathcal{H}_{Y}=$ $\left(Y, \mathcal{E}_{Y}\right)$, where

$$
\mathcal{E}_{Y}=\left\{E_{i} \cap Y, \quad E_{i} \in \mathcal{E} ; E_{i} \cap Y \neq \emptyset\right\}
$$

Definition 2.3. The equivalent edge number of $\mathcal{H}$ is the sum

$$
\mu(\mathcal{H})=\sum_{i \in I}\left(\left|E_{i}\right|-1\right)
$$



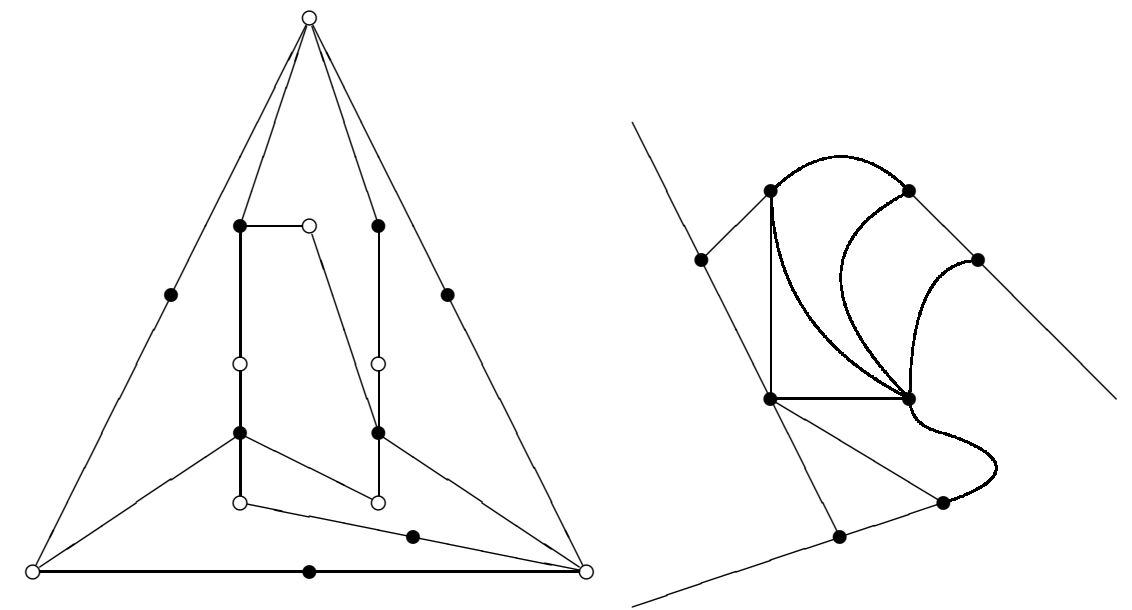

FiguRE 5. The incidence graph $\operatorname{Incid}(\mathcal{H})$ of a planar hypergraph $\mathcal{H}$ and a strong contact representation of $\mathcal{H}$

Definition 2.4. The incidence graph $\operatorname{Incid}(\mathcal{H})$ of $\mathcal{H}$ is the colored bipartite graph on $(X, \mathcal{E})$ defined by the vertex-edge incidence, a vertex being colored white (resp. black) if it belongs to $X$ (resp. $\mathcal{E}$ ).

As a special case, if $G$ is a graph, $\operatorname{Incid}(G)$ is the bicolored vertex-edge incidence graph of $G$.

Definition 2.5. A hypergraph $\mathcal{H}$ is planar if $\operatorname{Incid}(\mathcal{H})$ is planar.

The definition of strong contact representation of graphs extends naturally to hypergraphs:

DeFinition 2.6. A hypergraph $\mathcal{H}$ has a strong contact representation if there exists a contact family $\mathcal{F}$, whose arc-point incidence is the vertex-edge incidence of $\mathcal{H}$, so that arcs represents vertices and points represent edges (see Fig. E).

\subsection{Frames.}

Definition 2.7. A frame is a pair $\Phi=(\Gamma, \Pi)$, where $\Gamma$ is a plane graph and $\Pi=\left(F_{1}, \ldots, F_{p}\right)$ is a partition of the edge set $E(\Gamma)$, such that each class is either a bounded 2-connected face of $\Gamma$ or a single edge.

A vertex $x \in V(\Gamma)$ is incident to a class $F_{i} \in \Pi$ if it is incident to at least one edge in $F_{i}$.

A frame $\Phi$ defines a hypergraph $\mathcal{H}_{\Phi}=(V(\Gamma), \mathcal{E})$, where $E_{i}$ is the set of the vertices incident to the class $F_{i}$ of $\Pi$. We shall say that $\Phi$ is a frame representation of $\mathcal{H}_{\Phi}$ (see Fig. (6).

Proposition 2.1. A hypergraph $\mathcal{H}=(X, \mathcal{E})$ is planar if and only if it has a frame representation.

Proof. The bijection between the embedded incidence graph of a planar hypergraph and frame representations is shown on Fig. 7:

- From the incidence graph of a planar hypergraph $\mathcal{H}$, each black vertex (corresponding to some $E_{i}$ ) is split into a $C_{k}$ (if its degree is $k>2$ ), a $K_{2}$ (if its degree is 2), or a loop (if its degree is 1), which edges forms a set $F_{i}$. Then, the black-white edges are contracted. The resulting graph together with the partition $\left(F_{1}, \ldots, F_{m}\right)$ is a frame representation of $\mathcal{H}$. 

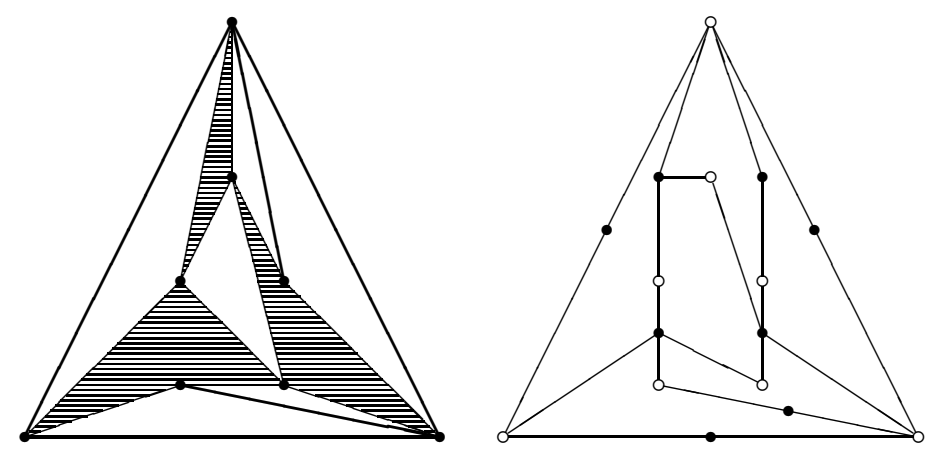

FiguRE 6. A frame representation of a planar hypergraph $\mathcal{H}$ and the incidence graph of $\mathcal{H}$ (the edges of $\mathcal{H}$ are the dashed faces and the thick line segments of the frame)
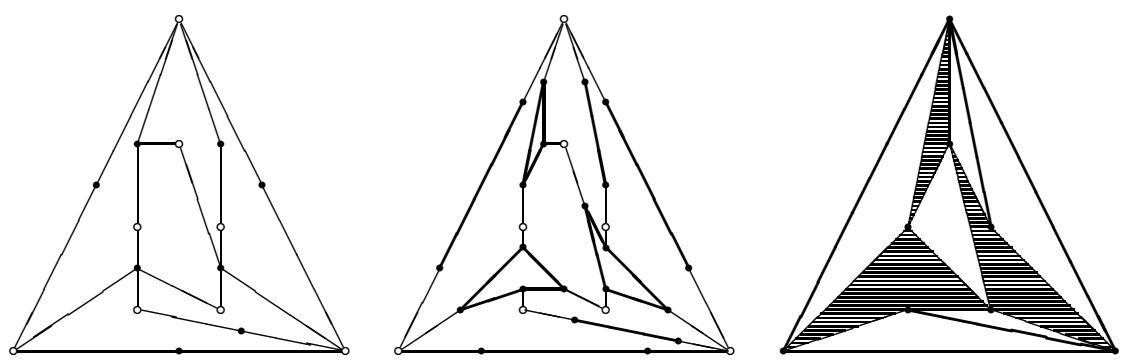

FIGURE 7. Bijection between incidence graphs of planar hypergraphs and frames

- From a frame $\Phi=(\Gamma, \Pi)$, we add a vertex in each face in $\Pi$ (including the faces defined by a loop) and join it to the vertices of the face, we bisect the non-loop edges that forms a single element class in $\Pi$ and then delete all the original edges of $\Gamma$ that have not been bisected.

REMARK 2.8. According to the definition of a linear hypergraph, the following conditions are equivalent:

- $\mathcal{H}$ is a linear planar hypergraph,

- $\mathcal{H}$ is a hypergraph which has a $C_{4}$-free frame representation,

- $\mathcal{H}$ is a planar hypergraph and all the frame representations of $\mathcal{H}$ are $C_{4^{-}}$ free.

\section{Strong Contact Representation}

\subsection{Strong contact representation of graphs.}

THEOREM 3.1. A graph $G$ has a strong contact representation if and only if it is planar and satisfies:

$$
\forall A \subseteq V, \quad\left|E\left(G_{A}\right)\right| \leq 2|A|
$$

Proof. Equation (3.1) is equivalent to the existence of a 2-orientation of $G$, that is an orientation of $G$ such that each vertex has at most 2 incoming edges. 


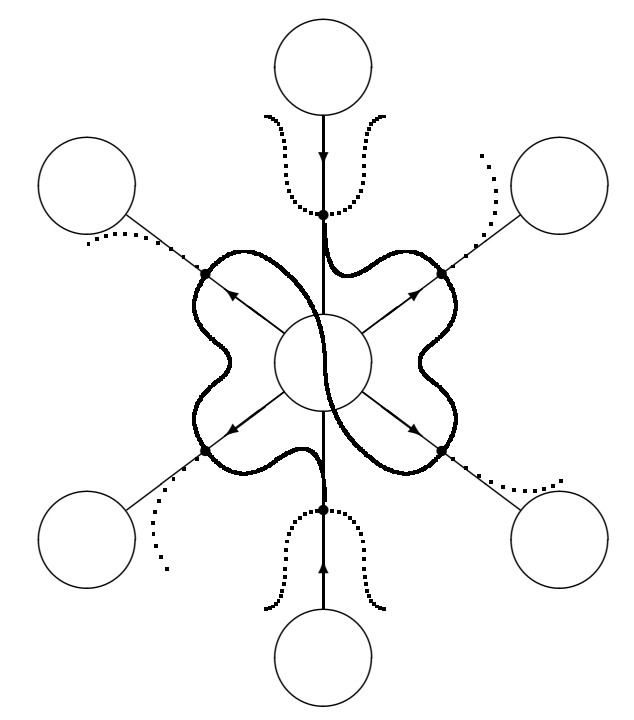

FiguRE 8. A contact representation induced by a 2-orientation

- A strong contact representation of a graph $G$ induces a planar embedding of $G$ and a 2-orientation of it: an edge incident to a vertex $v$ is oriented from $v$ if it corresponds to an internal point of the $\operatorname{arc} \nu$ representing $v$; edges corresponding to point which are internal to no arc are oriented arbitrarly.

- Conversely, an embedding and a 2-orientation of $G$ defines a strong contact representation of $G$ (see Fig. 8).

REMARK 3.2. This theorem implies that outer-planar graphs and triangle free planar graphs have a strong contact representation. Actually, bipartite planar graphs have a strong contact representation using segments in two directions [2].

3.2. Strong contact representation of hypergraphs. We may now generalize this result to hypergraphs. That for, we need first to prove an orientation lemma:

Lemma 3.3. The incidence graph $\operatorname{Incid}(\mathcal{H})$ of an hypergraph $\mathcal{H}$ has a 2-1 orientation, that is an orientation such that each white (resp. black) vertex has at most 2 (resp. 1) incoming edges, if and only if it satisfies

$$
\forall A \subseteq X, \quad \mu\left(\mathcal{H}_{A}\right) \leq 2|A|
$$

Proof. By classical arguments, $\operatorname{Incid}(\mathcal{H})$ has a 2-1 orientation if and only if it satisfies:

$$
\forall Y \subseteq X \cup \mathcal{E}, \quad\left|E\left(\operatorname{Incid}(\mathcal{H})_{Y}\right)\right| \leq 2|Y \cap X|+|Y \cap \mathcal{E}|
$$

That is:

$$
\forall A \subseteq X, \forall \mathcal{E}^{\prime} \subseteq \mathcal{E}, \quad\left|E\left(\operatorname{Incid}(\mathcal{H})_{A \cup \mathcal{E}^{\prime}}\right)\right| \leq 2|A|+\left|\mathcal{E}^{\prime}\right|
$$




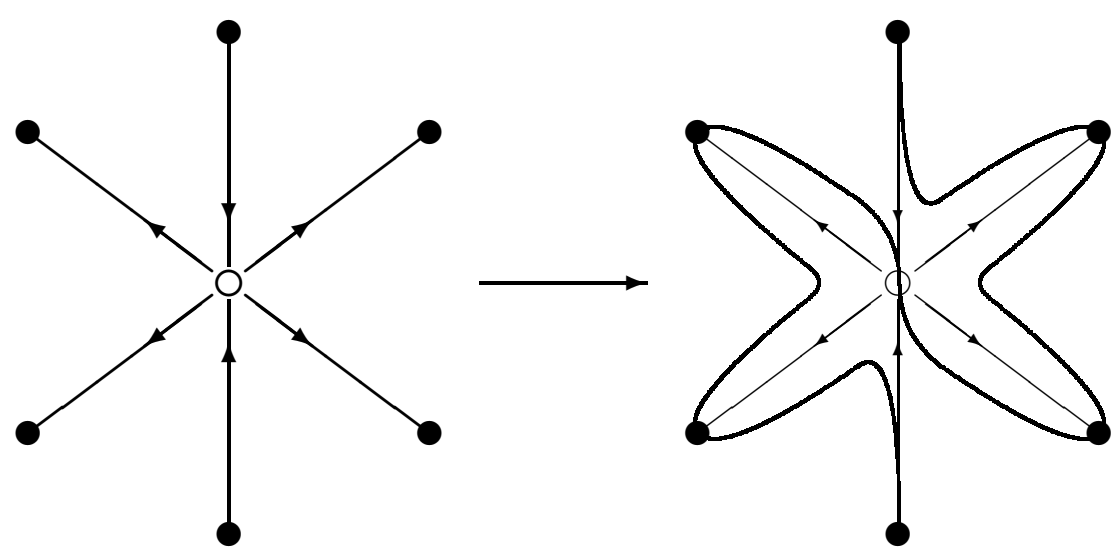

FigURE 9. Representation of the 2-oriented planar hypergraph from a 2-1 orientation of its incidence graph

It is obviously sufficient to consider pairs $\left(A, \mathcal{E}^{\prime}\right)$ where $\mathcal{E}^{\prime}$ is the neighbor set $N(A)$ of $A$. Hence, (3.4) may be rewritten:

$$
\forall A \subseteq X, \quad\left|E\left(\operatorname{Incid}(\mathcal{H})_{A \cup N(A)}\right)\right| \leq 2|A|+|N(A)|
$$

Finally,

$$
\begin{aligned}
\left|E\left(\operatorname{Incid}(\mathcal{H})_{A \cup N(A)}\right)\right|-|N(A)| & =\sum_{E \in N(A)} d_{\operatorname{Incid}(\mathcal{H})_{A \cup N(A)}(E)-|N(A)|} \\
& =\sum_{E \in N(A)}(|E \cap A|-1) \\
& =\mu\left(\mathcal{H}_{A}\right)
\end{aligned}
$$

Hence, the graph $\operatorname{Incid}(\mathcal{H})$ has a $2-1$ orientation if and only if $(3.2)$ holds.

THEOREM 3.4. A hypergraph $\mathcal{H}$ has a strong contact representation if and only if $\mathcal{H}$ is planar and satisfies

$$
\forall A \subseteq X, \quad \mu\left(\mathcal{H}_{A}\right) \leq 2|A|
$$

Proof. - A strong contact representation of a hypergraph $\mathcal{H}$ induces a planar embedding and a 2-1 orientation of $\operatorname{Incid}(\mathcal{H})$ : an edge $\{v, E\}$ is oriented from $v$ to $E$ if the point representing $E$ is interior to the arc representing $v$.

- Conversely, if $\mathcal{H}$ is a planar hypergraph, an embedding and a 2-1 orientation of $\operatorname{Incid}(\mathcal{H})$ defines a strong contact representation of $\mathcal{H}$ (see Fig. 9).

The theorem now follows from Lemma 3.3 .

3.3. Strong contact representation of frame-represented hypergraphs. We introduce some definitions and notations related to triangulation:

Let $\mathcal{H}$ be a planar hypergraph and $\Phi=(\Gamma, \Pi)$ be a frame representation of $\mathcal{H}$.

- Let $C$ be a polygon, then Triang $(C)$ is the set of the maximal outer-planar graphs which may be obtained by triangulating the inside of $C$.

- Let $E$ be an edge of $\mathcal{H}$. Then $\operatorname{Triang}(E)=\{E\}$ if $E$ as cardinality at most two and Triang $(E)=\operatorname{Triang}(C)$ if $C$ is the polygon of $\Gamma$ induced by the edges of the class of $\Pi$ corresponding to the edge $E$ of $\mathcal{H}$.

- Triang $(\Phi)$ is the set of the plane multigraphs which may be obtained from $\Gamma$ by triangulating the faces belonging to $\Pi$. 
- For any plane graph $G \in \operatorname{Triang}(\Phi), \mathrm{T}_{\Phi}(G)$ denotes the union of the set of all the triangular faces of $G$ which are not faces of $\Gamma$ and the set of all the triangular faces of $\Gamma$ which belong to $\Pi$.

For any induced plane subgraph $H$ of a plane graph $G \in \operatorname{Triang}(\Phi)$, $\mathrm{T}_{\Phi}(H)$ is the set of all the triangular faces of $H$ which belong to $\mathrm{T}_{\Phi}(G)$. Notice that this set does not depend on the choice of $G$ in Triang $(\Phi)$.

LEMma 3.5. $\mathcal{H}$ is a linear hypergraph if and only if all the graphs in $\operatorname{Triang}(\Phi)$ are simple.

Proof. - If $\mathcal{H}$ is not linear, there exists two edges $E, E^{\prime}$ of $\mathcal{H}$ having at least two vertices $x$ and $y$ in common. Hence, there exists a multigraph in Triang $(\Phi)$ such that $x$ an $y$ are linked by an edge in Triang $(E)$ and by an edge induced in Triang $\left(E^{\prime}\right)$.

- If $\mathcal{H}$ is linear, two vertices may simultaneously belong to at most one edge of $\mathcal{H}$ and hence are linked by at most one edge in a graph belonging to $\operatorname{Triang}(\Phi)$.

Lemma 3.6. Let $C$ be a polygon. Then, for any subset $W \subseteq V(C)$, we have:

$$
\max _{G \in \operatorname{Triang}(C)}\left|E\left(G_{W}\right)\right|-\left|T\left(G_{W}\right)\right|= \begin{cases}|W|-1 & \text { if } W \neq \emptyset \\ 0 & \text { otherwise }\end{cases}
$$

Proof. The number $\left|T\left(G_{W}\right)\right|$ of bounded triangular faces of $G_{W}$ is one less than the number of faces of $G_{W}$. Hence, $\left|E\left(G_{W}\right)\right|-\left|T\left(G_{W}\right)\right|$ is equal to $|W|-$ $c\left(G_{W}\right)$, where $c\left(G_{W}\right)$ is the number of connected components of $G_{W}$. If $W$ is nonempty, this number is at most $|W|-1$, and this value is achieved by any triangulation $G$ of $C$, such that $G_{W}$ is connected.

Lemma 3.7. Let $\Phi=(\Gamma, \Pi)$ be a frame representation of a hypergraph $\mathcal{H}$. Let $W \subseteq V$ be a subset of the vertex set of $\Gamma$. Then,

$$
\mu\left(\mathcal{H}_{W}\right)=\max _{G \in \operatorname{Triang}(\Phi)}\left(\left|E\left(G_{W}\right)\right|-\left|\mathrm{T}_{\Phi}\left(G_{W}\right)\right|\right)
$$

Proof. Let $\Gamma_{i}$ be the partial graph of $\Gamma$ induced by (edges of) $F_{i} \in \Pi$. Let $W_{i}$ be the subset of $W$ of the vertices incident to $F_{i}$. Any graph $G \in \operatorname{Triang}(\Phi)$ is uniquely defined by the list of the graphs $G^{(i)} \in \operatorname{Triang}\left(\Gamma_{i}\right)$.

The number $\left|E\left(G_{W}\right)\right|$ of edges of the subgraph of $G$ induced by $W$ is the sum of the numbers $\left|E\left(G_{W_{i}}^{(i)}\right)\right|$ of edges of the subgraphs of the $G^{(i)}$ induced by $W_{i}$.

Similarly, the number $\left|\mathrm{T}_{\Phi}\left(G_{W}\right)\right|$ is the sum of the numbers $\left|T\left(G_{W_{i}}^{(i)}\right)\right|$ of triangles of the subgraphs of the $G^{(i)}$ induced by $W_{i}$, as a triangle belongs to $\mathrm{T}_{\Phi}\left(G_{W}\right)$ if and only if its vertices belongs to the same $\Gamma_{i}$.

Hence,

$$
\begin{aligned}
\mu\left(\mathcal{H}_{W}\right) & =\sum_{i, W_{i} \neq \emptyset}\left(\left|W_{i}\right|-1\right) \\
& =\sum_{i}\left(\max _{H \in \operatorname{Triang}\left(\Gamma_{i}\right)}\left(\left|E\left(H_{W_{i}}\right)\right|-\left|T\left(H_{W_{i}}\right)\right|\right)\right) \\
& =\max \left\{\sum_{i}\left(\left|E\left(G_{W_{i}}^{(i)}\right)\right|-\left|T\left(G_{W_{i}}^{(i)}\right)\right|\right), \quad \forall j, G^{(j)} \in \operatorname{Triang}\left(\Gamma_{j}\right)\right\} \\
& =\max _{G \in \operatorname{Triang}(\Phi)}\left(\left|E\left(G_{W}\right)\right|-\left|\mathrm{T}_{\Phi}\left(G_{W}\right)\right|\right)
\end{aligned}
$$




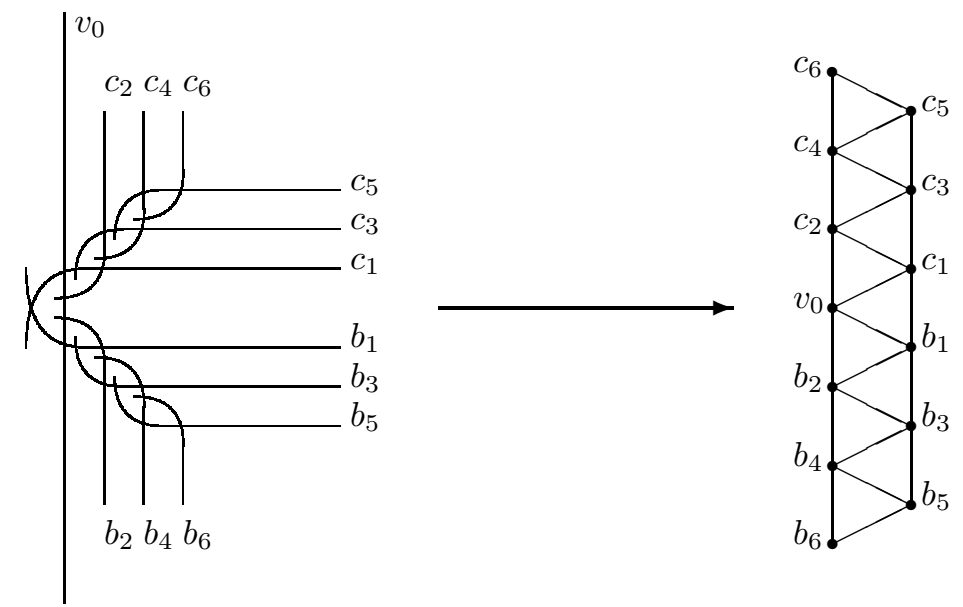

Figure 10. The local deformation of a contact point representing a zig-zag graph

THEOREM 3.8. Let $\Phi=(\Gamma, \Pi)$ be a frame representation of a plane linear hypergraph $\mathcal{H}$. Then, $\mathcal{H}$ has a strong contact representation if and only if the following inequality holds:

$$
\max _{G \in \operatorname{Triang}(\Phi), W \subseteq V(\Gamma)}\left|E\left(G_{W}\right)\right|-\left|\mathrm{T}_{\Phi}\left(G_{W}\right)\right|-2|W| \leq 0
$$

Proof. This is a direct consequence of Theorem 3.4 and Lemma 3.7.

\section{Local Intersections}

Let $H$ be a maximal outer-planar graph and let $\mathcal{F}$ be a family of $|V(H)| \operatorname{arcs}$ which share a single contact point $p$.

The graph $H$ is a local intersection graph if, for any vertex $v$ of $H$, there exists a local deformation of $\mathcal{F}$ at $p$ which represents $H$, and such that

- the arc to which $p$ is interior represents $v$,

- the circular order of the arcs is the same as the circular order of the vertices they represent.

A zig-zag graph is a maximal outer-planar graph which interior edges defines a path.

Lemma 4.1. A zig-zag graph is a local intersection graph.

Proof. The construction of a local deformation of a contact point into a zigzag graph is shown on Fig. 10.

REMARK 4.2. One can prove the reverse, that is: a maximal outer-planar graph is a local intersection graph if and only if it is a zig-zag graph.

Let $\mathcal{Z}(\Phi)$ denotes the set of all possible graphs obtained from $\Gamma$ by triangulating the faces in $\Pi$ using paths, and let $K(\Phi)$ be the graph obtained from $\Gamma$ by replacing each face in $\Pi$ by a clique.

THEOREM 4.3. Let $\Phi=(\Gamma, \Pi)$ be a frame.

If the following condition holds:

$$
\max _{G \in \operatorname{Triang}(\Phi), W \subseteq V(\Gamma)}\left|E\left(G_{W}\right)\right|-\left|\mathrm{T}_{\Phi}\left(G_{W}\right)\right|-2|W| \leq 0
$$

then: 

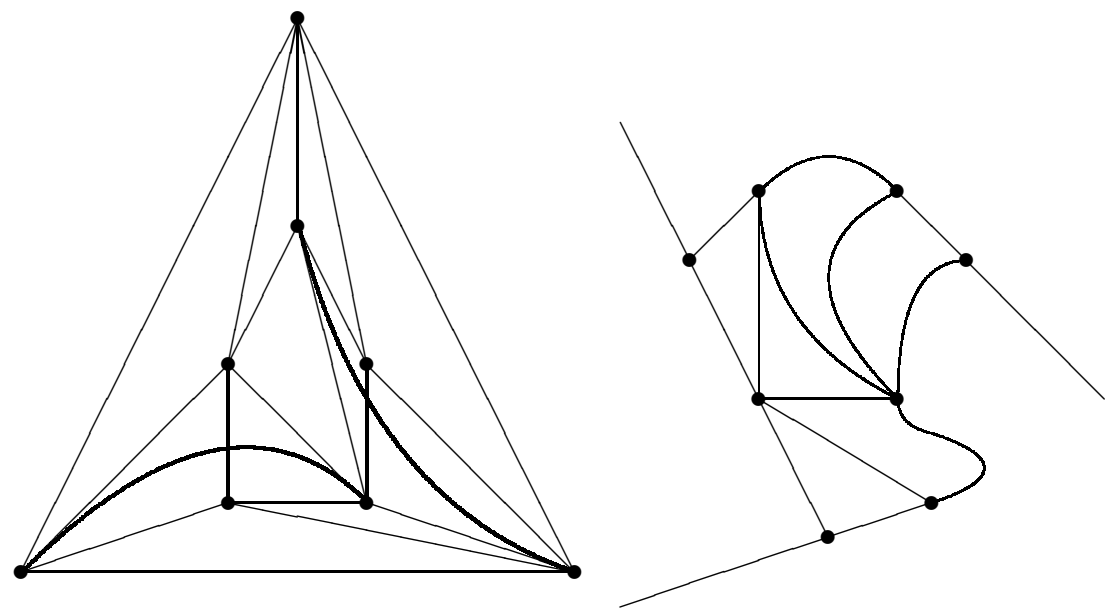

Figure 11. A contact representation of $K(\Phi)$
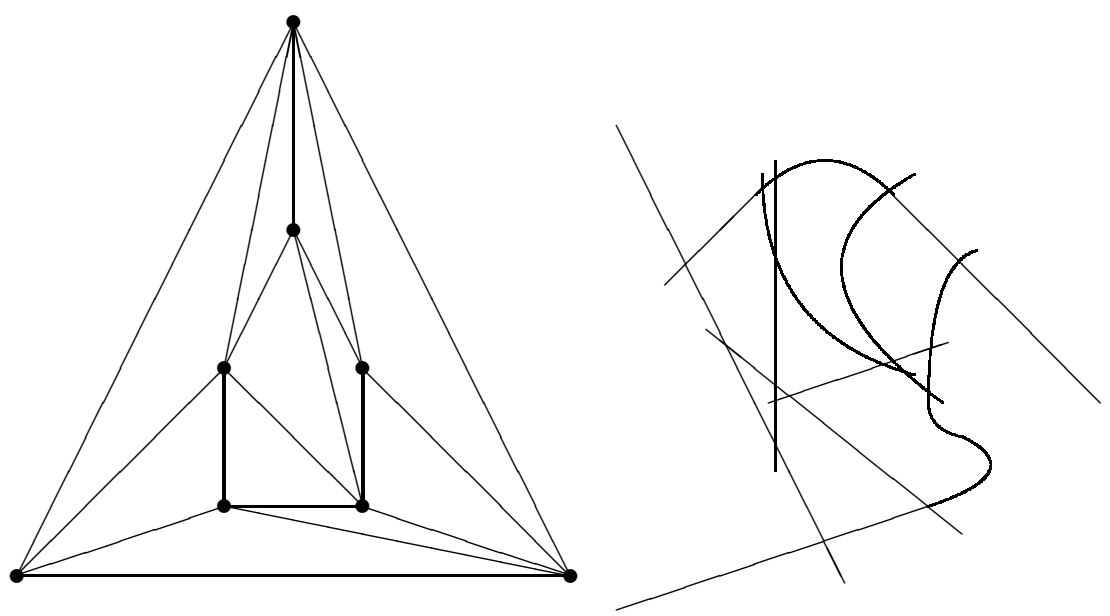

FIGURE 12. A strong intersection representation of a graph $G \in \mathcal{Z}(\Phi)$

- $K(\Phi)$ has a contact representation,

- any graph in $\mathcal{Z}(\Phi)$ has an intersection representation.

Proof. The conclusion follows from the previous lemma and Theorem 3.8 .

Corollary 4.3.1. Let $\Gamma(V, E)$ be a plane graph and let $\mathcal{I}$ be an independent (i.e. edge-disjoint) set of triangular faces (defining $\Pi=\mathcal{I} \cup\left\{\{e\}, \quad e \in E \backslash\left(\bigcup_{T \in \mathcal{I}} T\right)\right\}$ ). If the following condition holds:

$$
\max _{W \subseteq V}\left|E\left(\Gamma_{W}\right)\right|-\left|T\left(\Gamma_{W}\right) \cap \mathcal{I}\right|-2|W| \leq 0
$$

then $\Gamma$ has a contact representation. 

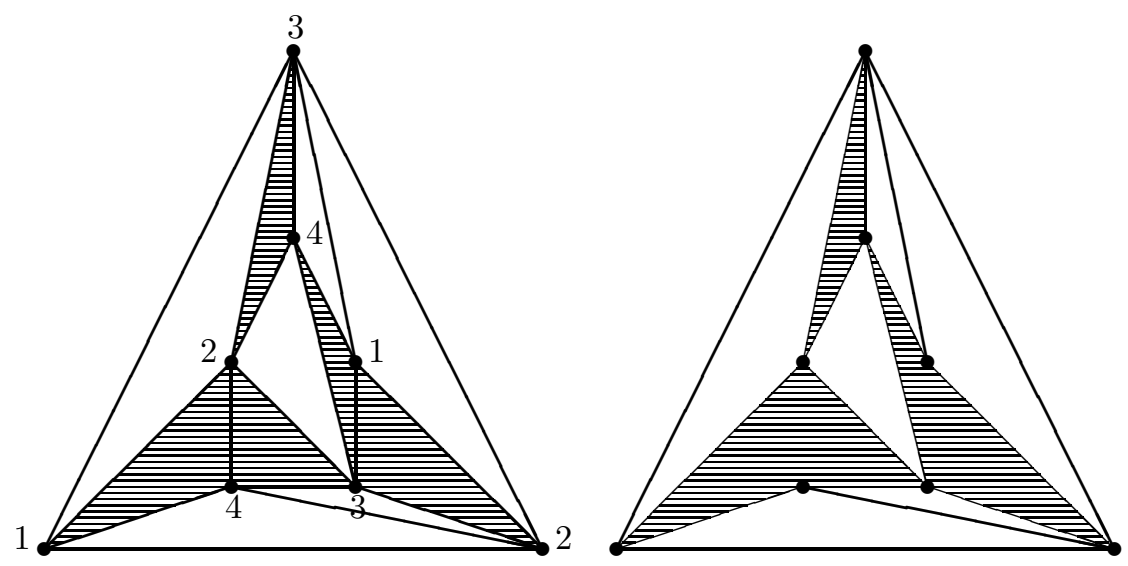

Figure 13. The 4-colored plane graph $G$ (the dashed triangles are the members of $\mathrm{T}^{+}(G)$ ) and the associated frame

\section{REPRESENTATION OF PLANAR GRAPHS}

\section{The Coloration Method}

Let $G$ be a 2-connected 4-colored plane graph. Let $\mathrm{T}^{+}(G)$ be the set of all the bounded triangular faces of $G$ in which two consecutive colors appear clockwise.

First notice that a triangle belonging to $\mathrm{T}^{+}(G)$ is adjacent to at most one other triangle belonging to $\mathrm{T}^{+}(G)$. More precisely, two bounded adjacent triangles both belong to $\mathrm{T}^{+}(G)$ if and only if the vertices of their union are colored $(1,2,3,4)$ in clockwise order.

A frame $\Phi=(\Gamma, \Pi)$ is obtained from $G$ and $\mathrm{T}^{+}(G)$ :

- The graph $\Gamma$ is the graph obtained from $G$ by erasing the edges belonging to two triangles belonging to $\mathrm{T}^{+}(G)$.

- The partition $\Pi$ has the following classes:

- $\{e\}$, if the edge $e$ belongs to no triangle in $\mathrm{T}^{+}(G)$,

$-T$, if the triangle $T$ belongs to $\mathrm{T}^{+}(G)$ and no triangle adjacent to it belongs to $\mathrm{T}^{+}(G)$,

- $\left(T_{1} \cup T_{2}\right) \backslash\left(T_{1} \cap T_{2}\right)$, if the triangles $T_{1}$ and $T_{2}$ are adjacent and both belong to $\mathrm{T}^{+}(G)$.

Lemma 5.1. If $G$ has no induced $C_{4}$ colored $(1,2,3,4)$ and no separating $C_{3}$ with two consecutive colors appearing clockwise, then all the graphs in $\operatorname{Triang}(\Phi)$ have the same property

Proof. All the graphs in $\operatorname{Triang}(\Phi)$ may be obtained from $G$ by a sequence of edge-switchings performed on edges not in $E(\Gamma)$.

If such a switching creates a $C_{3}$ separator with two consecutive colors appearing clockwise (say $v_{3}, v_{1}, v_{2}$, where the edge $\left(v_{3}, v_{1}\right)$ comes from the switching of an edge $\left.\left(x_{2}, x_{4}\right)\right)$, then the original graph has $\left(v_{3}, x_{4}, v_{1}, v_{2}\right)$ as an induced $C_{4}$.

Similarly a $C_{3}$ separator with two consecutive colors appearing clockwise may only occur if an induced $C_{4}$ colored $(1,2,3,4)$ exists before the edge-switching.

Lemma 5.2. For any $G \in \operatorname{Triang}(\Phi)$, and for any induced subgraph $H \subseteq G$, we have:

$$
\mathrm{T}^{+}(H)=\mathrm{T}_{\Phi}(H)
$$


ProOF. This is a direct consequence of the fact that there exists no $C_{3}$ separator with two consecutive colors appearing clockwise in $G$.

Lemma 5.3. If $G$ has no induced $C_{4}$ colored $(1,2,3,4)$ in the clockwise order, we have:

$$
|E(G)|-\left|\mathrm{T}^{+}(G)\right|-2|V(G)| \leq-3
$$

PROOF. Let us prove the lemma by induction on the size sequence of the faces.

A graph is minimal if each face has length 3 and hence is a maximal planar graph. Then, any triangle of $G$ includes exactly one edge colored $(1,2)$ or $(3,4)$ and such an edge belongs to two adjacent triangles. This matching shows that $\mathrm{T}^{+}(G)$ includes either the half of the triangles of $G$ or the half the triangles of $G$ number minus 1 (depending on the coloration of the outer face which never belongs to $\left.T^{+}(G)\right)$. Hence:

$$
\begin{aligned}
|E(G)|-\left|\mathrm{T}^{+}(G)\right|-2|V(G)| & \leq(3|V(G)|-6)-\left(\frac{1}{2}(2|V(G)|-4)+1\right)-2|V(G)| \\
& \leq-3
\end{aligned}
$$

Otherwise, we shall construct a 4-colored 2-connected plane graph $G^{\prime}$ with no face of length 4 colored $(1,2,3,4)$ in the clockwise order that satisfies (together with the associated subset $\mathrm{T}^{+}\left(G^{\prime}\right)$ of its face set):

$$
\left|E\left(G^{\prime}\right)\right|-\left|\mathrm{T}^{+}\left(G^{\prime}\right)\right|-2\left|V\left(G^{\prime}\right)\right| \geq|E(G)|-\left|\mathrm{T}^{+}(G)\right|-2|V(G)|
$$

Let $F$ be a non-triangular face of $G$ of maximal size. We shall consider several cases:

- There exists a color $c$, such that 4 vertices of $F$ at least are not colored $c$.

Then $G^{\prime}$ is obtained from $G$ by adding a vertex $x$ in $F$ colored $c$, which is linked to all the $k \geq 4$ vertices of $F$, which are not colored $c$. We get:

$$
\begin{aligned}
\left|V\left(G^{\prime}\right)\right| & =|V(G)|+1 \\
\left|E\left(G^{\prime}\right)\right| & =|E(G)|+k \\
\left|\mathrm{~T}^{+}\left(G^{\prime}\right)\right| & \leq|T(G)|+\left\lfloor\frac{2 k}{3}\right\rfloor
\end{aligned}
$$

Thus,

$$
\begin{aligned}
\left|E\left(G^{\prime}\right)\right|-\left|\mathrm{T}^{+}\left(G^{\prime}\right)\right|-2\left|V\left(G^{\prime}\right)\right| & \geq\left\lceil\frac{k}{3}\right\rceil-2+|E(G)|-\left|\mathrm{T}^{+}(G)\right|-2|V(G)| \\
& \geq|E(G)|-\left|\mathrm{T}^{+}(G)\right|-2|V(G)|
\end{aligned}
$$

Remark that no face of length 4 colored $(1,2,3,4)$ in clockwise order may be created that way.

- Otherwise, no color is present twice on $F$. If $V_{i}(F)$ denotes the set of the $i$-colored vertices of $F$ and $l$ denotes the length of $F$, then:

$$
4 \leq l=\left|\bigcup_{i} V_{i}(F)\right|=\frac{1}{3} \sum_{i}\left|\bigcup_{j \neq i} V_{j}(F)\right| \leq 4
$$

Hence, $F$ has length 4 .

We triangulate $F$ by adding an edge and, as $F$ is not colored $(1,2,3,4)$ in clockwise order, at most one additional triangle is added to $\mathrm{T}^{+}\left(G^{\prime}\right)$. Hence,

$$
\left|E\left(G^{\prime}\right)\right|-\left|\mathrm{T}^{+}\left(G^{\prime}\right)\right|-2\left|V\left(G^{\prime}\right)\right| \geq|E(G)|-\left|\mathrm{T}^{+}(G)\right|-2|V(G)|
$$


Lemma 5.4. If $G$ has no induced $C_{4}$ colored $(1,2,3,4)$ in the clockwise order and no separating $C_{3}$ with two consecutive colors appearing clockwise, we have:

$$
\max _{W \subseteq V(G)}\left|E\left(G_{W}\right)\right|-\left|\mathrm{T}^{+}\left(G_{W}\right)\right|-2\left|V\left(G_{W}\right)\right| \leq-3
$$

Proof. Let us first prove that the inequality

$$
\left|E\left(G_{W}\right)\right|-\left|\mathrm{T}^{+}\left(G_{W}\right)\right|-2\left|V\left(G_{W}\right)\right| \leq-3
$$

holds for any induced 2-connected subgraph $H \subseteq G$ in place of $G_{W}$.

Actually, let $\mathrm{T}^{+}(H)$ be the set of all the bounded triangles of $H$ in which two consecutive colors appear clockwise. As $G$ has no separating $C_{3}$ with two consecutive colors appearing clockwise, $\mathrm{T}^{+}(H)=T(H) \cap \mathrm{T}^{+}(G)$. Moreover, $H$ has no face of length 4 colored $(1,2,3,4)$ in clockwise order as it would be an induced 4-cycle of $G$.

Hence, by the preceding lemma, any 2-connected induced subgraph $H$ of $G$ satisfies:

$$
|E(H)|-\left|\mathrm{T}^{+}(H)\right|-2|V(H)| \leq-3
$$

¿From this inequality, we deduce that the same holds for any induced subgraph $H$ of $G$ (remark that the weaker inequality $|E(H)|-\left|\mathrm{T}^{+}(H)\right|-2|V(H)| \leq 0$ could not be extended to not 2-connected graphs).

THEOREM 5.5. Let $G$ be a 4-colored 2-connected plane graph with no induced 4-cycle colored $(1,2,3,4)$ in the clockwise order and no separating $C_{3}$ with two consecutive colors appearing clockwise.

Then, $G$ has an intersection representation.

Proof. ¿From the previous lemmas, we get

$$
\max _{G \in \operatorname{Triang}(\Phi), W \subseteq V(\Gamma)}\left|E\left(G_{W}\right)\right|-\left|\mathrm{T}_{\Phi}\left(G_{W}\right)\right|-2|W| \leq-3
$$

and, as $G$ belongs to $\mathcal{Z}(\Phi)=\operatorname{Triang}(\Phi)$, the results follows.

Corollary 5.5.1. Let $G$ be an internally 5-connected planar graph. Then, $G$ has an intersection representation.

THEOREM 5.6. Let $G$ be a 3-colored 2-connected plane graph with no separating $C_{3}$ having two consecutive colors appearing clockwise,

Then, $G$ has a contact representation.

Proof. As previously, we get

$$
\max _{G \in \operatorname{Triang}(\Phi), W \subseteq V(\Gamma)}\left|E\left(G_{W}\right)\right|-\left|\mathrm{T}_{\Phi}\left(G_{W}\right)\right|-2|W| \leq-3
$$

and, as $G$ is equal to $K(\Phi)$, the results follows.

Corollary 5.6.1. Let $G$ be a 4-connected 3-colorable planar graph. Then, $G$ has a contact representation.

\section{Cut and Paste}

By cutting $C_{3}$-separated components and pasting back their representation we obtain the following extension of Theorem 5.5:

TheOREM 6.1. Let $G$ be a 4-colored plane graph with no induced 4-cycle colored $(1,2,3,4)$ in the clockwise order.

Then, $G$ has an intersection representation. 


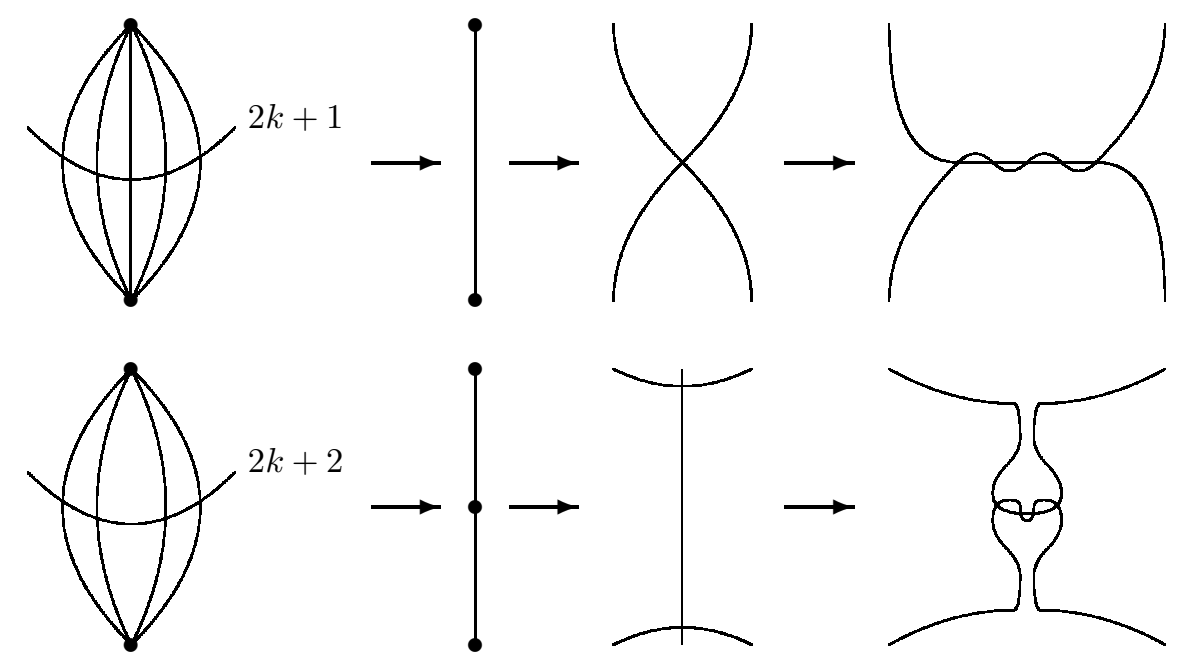

FIGURE 14. The existence of a strong intersection representation for all simple planar graphs is equivalent to the existence of a strong intersection representation for all planar multigraphs

\section{Conclusion}

Here we consider general graphs and hypergraphs (with possible loops and multiple edges).

Definition 7.1. A hypergraph $\mathcal{H}$ (or a multigraph $G$ ) has a strong intersection representation if there exists a family $\mathcal{F}$ of Jordan arcs, whose arc-point incidence is the vertex-edge incidence of $\mathcal{H}$ (or $G$ ): arcs represents vertices and points represent edges.

Problem 1. Has any planar simple graph an intersection representation?

Problem 2. Has any planar multigraph a strong intersection representation?

Proposition 7.1. The problems 1 and 目are equivalent:

Any planar simple graph has an intersection representation if and only if any planar multigraph has a strong intersection representation.

Proof. If any planar multigraph has a strong intersection representation, any planar simple graph has indeed an intersection representation.

Conversely, if any planar simple graph has an intersection representation, any planar simple graph has also a strong intersection representation obtained by a trivial local deformation of the arcs at the intersection points. If a planar multigraph $G$ has multiple edges, we consider the planar simple graph $G^{\prime}$ obtained from $G$ by replacing any edge set $E_{i}$ linking two vertices $x$ and $y$ by:

- a single edge $\{x, y\}$ if $\left|E_{i}\right|$ is odd,

- a bisected edge $\{x, z\},\{z, y\}$ if $\left|E_{i}\right|$ is even.

Then, a strong intersection representation of $G^{\prime}$ induces a strong intersection representation of $G$ (see Fig. 14).

Problem 3. Has any planar hypergraph a strong intersection representation?

Acknowledgment 1. The authors would like thank R. Šámal for his helpful comments and remarks. 


\section{References}

[1] C. Berge, Graphes et hypergraphes, second ed., Dunod, Paris, 1973.

[2] H. de Fraysseix, P. Ossona de Mendez, and J. Pach, Representation of planar graphs by segments, Intuitive Geometry 63 (1991), 109-117.

[3] G. Ehrlich, S. Even, and R.E. Tarjan, Intersection graphs of curves in the plane, Journal of Combinatorial Theory 21(B) (1986), 8-20.

[4] András Frank, On the orientation of graphs, Journal of Combinatorial Theory, Series B 28 (1980), 251-261.

[5] H. de Fraysseix, T. Matsumoto, P. Ossona de Mendez, and P. Rosenstiehl, Regular orientations and graph drawing, Abstracts for the Third Slovenian international conference in graph theory, 1995, pp. 12-13.

[6] H. de Fraysseix and P. Ossona de Mendez, On Regular Orientations, Prague Midsummer Combinatorial Workshop, 1994, abstract, pp. 9-13.

[7] _ Intersection graphs of Jordan arcs, Discrete and Computational Geometry : Ten Years Later, 1995, p. 14.

[8] S.L. Hakimi, On the degree of the vertices of a directed graph, J. Franklin Inst. 279 (1965), 4.

[9] J. Kratochvíl, A special planar satisfiability problem and a consequence of its NPcompleteness, Discrete Applied Mathematics 52 (1994), 233-252.

[10] J. Kratochvíl and J. Matoušek, Intersection graphs of segments, Journal of Combinatorial Theory 62(B) (1994), no. 2, 289-315.

[11] _ Are string graph algorithmically recognizable ?, Problem Session of the conference Graph and Geometry, Prague, 1997.

Patrice Ossona de Mendez, CNRS UMR 0017, E.H.E.S.S., 54 Bd Raspail, 75006 Paris, FRANCE

E-mail address: pom@ehess.fr

Hubert de Fraysseix, CNRS UMR 0017, E.H.E.S.S., 54 Bd Raspail, 75006 Paris, FRANCE

E-mail address: hf@ehess.fr 